Projets

de paysage

\section{Projets de paysage}

Revue scientifique sur la conception et l'aménagement de l'espace

19 | 2018

L'imagerie du paysage

\title{
Entre campagne publicitaire et films d'auteur : douze paysages urbains italiens au cinéma
}

Between the Advertising Campaign and the Authored Film: Twelve Italian Urban Landscapes in Cinema

\section{Olivier Gaudin}

\section{(2) OpenEdition}

\section{Journals}

Édition électronique

URL : https://journals.openedition.org/paysage/451

DOI : $10.4000 /$ paysage.451

ISSN : 1969-6124

Éditeur :

École nationale supérieure du paysage de Versailles-Marseille, Institut national des sciences appliquées Centre Val de Loire - École de la nature et du paysage, École nationale supérieure d'architecture et de paysage de Bordeaux, École nationale supérieure d'architecture et de paysage de Lille, Agrocampus Angers

\section{Référence électronique}

Olivier Gaudin, « Entre campagne publicitaire et films d'auteur : douze paysages urbains italiens au cinéma », Projets de paysage [En ligne], 19 | 2018, mis en ligne le 01 décembre 2018, consulté le 21 septembre 2021. URL : http://journals.openedition.org/paysage/451 ; DOI : https://doi.org/10.4000/ paysage. 451

Ce document a été généré automatiquement le 21 septembre 2021.

\section{c) (†)}

La revue Projets de paysage est mise à disposition selon les termes de la Licence Creative Commons Attribution - Pas d'Utilisation Commerciale - Pas de Modification 4.0 International. 


\section{Entre campagne publicitaire et films d'auteur : douze paysages urbains italiens au cinéma}

Between the Advertising Campaign and the Authored Film: Twelve Italian Urban Landscapes in Cinema

Olivier Gaudin

\section{NOTE DE L'AUTEUR}

Dodici registi per dodici città, films de Michelangelo Antonioni, Bernardo et Giuseppe Bertolucci, Mauro Bolognini, Alberto Lattuada, Carlo Lizzani, Mario Monicelli, Ermanno Olmi, Gillo Pontecorvo, Francesco Rosi, Mario Soldati, Lina Wertmüller et Franco Zeffirelli, prod. Istituto Luce/ministerio del Turismo e del Spettacolo, Italie, 1989, 90 minutes.

Note : les films sont disponibles en ligne sur l'ancienne chaîne Youtube de l'Istituto Luce, en quatre parties : https://www.youtube.com/channel/ UCl8gGyVItv8CBPt5r3qn3Gg (consulté le 20 septembre 2018).

Une nouvelle version de cette page semble mettre en ligne progressivement des versions en haute définition des films. Voir également le site http:// www.cinecittaluce.it/.

1 En 1989, en préparation de la $14^{\mathrm{e}}$ Coupe du monde de football organisée en Italie (Italia '90), le ministère italien du Tourisme et du Spectacle commande une série de courtsmétrages promotionnels à l'Institut Luce, qui s'adresse lui-même à douze réalisateurs de renom. Il s'agit de dresser de brefs portraits des douze villes accueillant les rencontres sportives, afin de composer un ensemble de séquences indépendantes. Le projet s'adresse en priorité aux touristes et supporteurs étrangers : Dodici Registi per dodici città (« Douze réalisateurs pour douze villes »), bien que produit par le prestigieux Istituto Luce, est d'abord un film publicitaire. On pourrait donc mettre en doute le 
statut d'œuvre d'art de ces douze séquences. Sauf que les cinéastes qui les ont réalisées - tous italiens - étaient aussi, sans exception, des artistes reconnus, voire consacrés : Michelangelo Antonioni (Roma), Bernardo et Giuseppe Bertolucci (Bologna), Mauro Bolognini (Palermo), Alberto Lattuada (Genova), Carlo Lizzani (Cagliari), Mario Monicelli (Verona), Ermanno Olmi (Milano), Gillo Pontecorvo (Udine), Francesco Rosi (Napoli), Mario Soldati (Torino), Lina Wertmüller (Bari) et Franco Zeffirelli (Firenze). Les réalisateurs ont visiblement disposé d'une marge d'interprétation, voire de reformulation, de la commande initiale - présenter chacune des villes accueillant l'événement sportif sous un angle favorable, voire ludique et festif -, au point d'en faire, dans certains cas au moins, de véritables petits films d'auteur. Si bien que l'ensemble offre un corpus exemplaire pour interroger le rapport entre la représentation cinématographique du paysage urbain et la rhétorique de l'imagerie publicitaire. Comment les villes sont-elles représentées? Que révèlent les choix esthétiques de ces mises en scène audiovisuelles de paysages urbains, qui suivent une logique de célébration à la fois promotionnelle, touristique et patrimoniale? Au fil d'une analyse comparée, le présent article examine les places respectives que ces films publicitaires accordent aux vues paysagères et aériennes, mais aussi aux monuments historiques ou aux caractéristiques géomorphologiques des sites (le relief, l'eau); ou encore, l'attention très variable que les différents cinéastes portent aux lieux publics, aux environnements sonores, aux activités ordinaires et aux habitants de ces villes.

2 À première vue, ces douze courts-métrages ne sont ni des œuvres documentaires ni des fictions. On peut néanmoins dire que la série de films joue en permanence sur la limite entre ces genres, une limite qui n'a jamais été une frontière et que le cinéma a rendue largement poreuse avec le néoréalisme italien (Bazin, 1975), la Nouvelle Vague française (Chabrol et al., 1999), ou encore les œuvres de Chris Marker, qui entretiennent un rapport si étroit à la documentation de l'histoire contemporaine (Jacques, 2018). Sans aller jusqu'à composer des scènes dialoguées, tous ces courts-métrages impliquent d'importants moyens et une préparation soignée des prises de vues, qui relèvent de l'industrie du cinéma la plus traditionnelle: des angles audacieux qui supposent des mouvements de caméra complexes; des éclairages savants; des moyens d'enregistrement, de composition et de mixage sonore professionnels et, bien entendu, un travail de montage très précis; voire, dans certains cas, une direction d'acteurs. Chacune des séquences utilise un large éventail des ressources techniques du cinéma de fiction, comme pour se démarquer avec soin d'une esthétique télévisuelle et conserver une légitimité artistique. L'emploi de ces procédés formels varie cependant de manière significative d'un auteur à l'autre; on verra en premier lieu que cette hétérogénéité s'observe avant tout sur le plan de la mise en récit, c'est-à-dire du contenu narratif des séquences, alors que les éléments formels des séquences sont similaires. Au terme de cette analyse comparative, je chercherai dans un second temps à ressaisir le sens de la série de films dans son ensemble, ce qui sera aussi l'occasion de revenir sur leur portée documentaire, plus prononcée dans certains cas. Je montrerai son originalité par rapport à la logique promotionnelle de l'imagerie qui s'est imposée depuis une trentaine d'années dans la représentation officielle des villes : un " marketing urbain » à vocation essentiellement touristique et marchande, dont les années 1990 ont été le laboratoire. 


\section{Des procédés formels comparables, des choix narratifs distincts}

\section{Les codes traditionnels de la représentation de la ville comme un paysage}

3 Les procédés de fabrication des films fournissent un premier objet d'analyse. Les séquences semblent partager un certain nombre de codes esthétiques et techniques généraux, sans doute en raison de la commande : les réalisateurs font tous un usage intensif de travellings et de musique, et adoptent un montage rapide où alternent des angles de vues plus ou moins larges, y compris plusieurs plans panoramiques. Ainsi, la présence de vues aériennes et paysagères où apparaît à distance l'horizon reconnaissable d'une skyline est systématique, et participe sans surprise d'une «banalisation » de la « consommation paysagère " (Debarbieux, 2007). Ce type d'images traduit en effet une attitude de rétrospection, voire de nostalgie et de fétichisme qui reproduit indéfiniment des formes déjà connues, correspondant aux attentes d'une majorité de visiteurs. D'une manière générale, les films étudiés reprennent des codes de représentation traditionnels que l'on peut rapporter à l'esthétique des vues urbaines de la Renaissance. Héritières de l'imagerie médiévale de la cité en tant que totalité organisée, elles la renouvelaient aussi en représentant chaque localité comme un ensemble unifié et cohérent inséré dans un milieu environnant productif et contrôlé. La plupart de ces images mettaient en évidence les accès depuis l'extérieur et les terres cultivées, ordonnées et entretenues avec soin, qui entouraient ces centres urbains. Par exemple, les vues de Bologne gravées et imprimées aux XVII ${ }^{e}$ et XVIII ${ }^{e}$ siècles (Ricci, 1980) montrent avec précision les portes, les canaux, les maraîchages et les vergers de part et d'autre de l'enceinte, et la topographie caractéristique des collines attenantes à la ville. Au-delà des légendes se rapportant aux principaux édifices et monuments numérotés, de tels portraits de cités contenaient des informations détaillées sur le paysage propre à chaque localité. Leur format et leur assemblage dans des ouvrages ont rendu possible leur circulation dans toute l'Europe et au-delà, au cours du XVIII siècle, ainsi que leur généralisation à des villes de taille et d'importance variables.

4 Très codifiées, ces images sont donc plutôt des portraits que des paysages: de véritables figurations personnifiées, fourmillant d'informations précises sur les formes bâties mais aussi sur le type d'activités, de circulations et de commerces qui caractérisent la ville représentée. On peut arrêter son regard sur des détails, parcourir l'image en suivant un itinéraire précis, ou bien embrasser l'ensemble d'un seul coup d'œil. Leur trait le plus marquant est d'insister sur l'organisation contrôlée de l'espace : toutes les villes sont représentées en temps de paix, dans un état d'équilibre, d'unité et de fonctionnement optimal, idéalisé et aseptisé. L'intention est d'attirer l'œil du spectateur sur des singularités visuelles remarquables qui fondent le prestige de la ville en même temps qu'elles favorisent la mémorisation et la reconnaissance. Préfigurant les paysages conventionnels des cartes postales, ces images cadrées avec soin composent une sorte d'abrégé de la ville concernée, sa miniaturisation graphique indéfiniment reproductible par les moyens de l'imprimerie.

5 Les représentations cinématographiques qui nous intéressent reprennent la filiation esthétique de cette imagerie traditionnelle, bien davantage que celle, par exemple, des «symphonies urbaines » de l'entre-deux-guerres ${ }^{1}$. Elles cherchent moins à documenter 
la vie sociale effective d'une ville - faite de tensions, de frictions et de divisions sociales et spatiales - qu'à en composer un visage, une façade ou un profil identifiable. Elles présentent ainsi une vitrine unifiée, cohérente et flatteuse à destination des visiteurs étrangers. Comme les portraits urbains du passé, les douze films de 1989 condensent un certain nombre de traits pittoresques et mémorables de chacune des douze villes. Rappelant parfois le court-métrage documentaire de Pasolini sur La Forma della città ${ }^{2}$, elles sont attentives à l'unité formelle et aux caractéristiques visibles les plus stables de ces paysages urbains, à leurs horizons reconnaissables; elles en recherchent les permanences, les propriétés organiques. La présence des monuments historiques, des façades ornées et des éléments de patrimoine y est donc centrale, au risque de négliger entièrement la réalité construite des environnements urbains de la fin du $\mathrm{XX}^{\mathrm{e}}$ siècle.

\section{Mettre en scène et animer le patrimoine urbain : une logique seulement marchande?}

6 À la différence des vues dessinées ou peintes, les séquences filmées utilisent sans réserve les moyens du cinéma - à commencer par le mouvement et le montage - afin de concentrer chaque portrait en un format bref d'une dizaine de minutes, qui n'excède pas la capacité d'attention d'un (télé)spectateur venu assister à des rencontres de football. L'une des raisons pour laquelle chacun des films insiste autant sur le mouvement est sans doute le contexte de la commande. Entre tourisme et spectacle, le public d'une Coupe du monde de football est en quête de sensations et d'émotions collectives, que l'on peut partager avec un grand nombre d'autres spectateurs. Mais n'est-ce pas là une spécialité de l'art du cinéma, qui fut longtemps un art de foire ou de foule avant d'accéder à une reconnaissance esthétique plus aboutie et au statut de " grand art »? L'industrie culturelle la plus populaire et la plus emblématique du siècle dernier - bien qu'elle ait connu un net recul avec l'avènement progressif de la télévision - n'a jamais renoncé à sa dimension initiale de divertissement en public, ouvert à une diffusion d'une ampleur sans précédent. Répondant à la commande officielle, les réalisateurs semblent donc adopter cette dimension de spectacle destiné au public le plus large: ils utilisent de manière appuyée et systématique les mouvements de caméra, des cadrages habiles, un montage rapide et une musique omniprésente.

7 À la fin des années 1980, le tourisme urbain était loin d'être aussi développé qu'aujourd'hui, en particulier sur le plan de la communication visuelle, qui a tant évolué au cours du dernier demi-siècle (Gervereau, 2003). Les affiches des agences de voyage et les guides touristiques en restaient alors les principaux vecteurs illustrés, avec la photographie, le cinéma et la télévision. Par contraste avec les usages numériques contemporains et la quantité exponentielle d'«images techniques» (Flusser, 2011) auxquelles nous sommes désormais surexposés, la diffusion et la circulation d'images en mouvement représentant des villes ou des paysages restaient limitées. Les films étudiés ici proviennent d'un autre âge de l'image. Ce contexte suggère que leur effort de mise en scène du patrimoine urbain était innovant: à l'exception notable du Roma d'Antonioni et du Palermo de Bolognini, qui se concentrent sur une mise en valeur exclusive de l'architecture, des statues et des palais, le spectateur peut admirer des lieux publics animés en même temps qu'un patrimoine fraîchement restauré. La mise en scène présente les monuments dans leur contexte urbain: le regard de la caméra se laisse guider par l'organisation spatiale des lieux 
centraux - en particulier les places et les axes principaux. Mais si l'on reconnaît les perspectives de l'espace florentin comme la piazza Santa Croce, ou la piazza del Duomo à Milan, on ne verra à l'écran ni la périphérie de Vérone striée d'axes routiers et ferroviaires, ni les banlieues encombrées de Rome, ni les collines qui entourent Florence, ni les abords industriels de Bologne et de Milan. La plupart des films donnent à voir les foyers d'intensité officiels de ces villes, c'est-à-dire leurs centres historiques, culturels et marchands. Ils en omettent presque tous les infrastructures, les secteurs de production et les quartiers d'habitation. La majorité des douze séquences compose donc des paysages urbains sélectifs et restreints, au risque d'une banalisation commerciale. Elles ne cherchent nullement à rejoindre le propos des chefs-d'œuvre d'Antonioni (L'Éclipse, 1962 ; Le Désert rouge, 1964), de Fellini (Fellini Roma, 1972) ou de Pasolini (Accattone, 1961; Mamma Roma, 1962), qui abordaient les paysages urbains et suburbains de manière directe, dans le sillage du néoréalisme. Conformément à la commande, ces petits films veulent plutôt faire percevoir au spectateur une ou plusieurs ambiances festives représentatives de la ville concernée: des attitudes reconnaissables et des lieux familiers. La démultiplication des mouvements de caméra et des angles de prises de vues (en particulier aériens ou de surplomb), tout comme la vitesse du montage visent sans doute à compenser le risque de répétition et d'ennui afin d'animer ces images et de les distinguer d'une simple série de cartes postales. Aux antipodes des usages quotidiens de leurs habitants, ces choix formels transforment les lieux centraux de ces villes plurimillénaires en simple décor, voire en objets de consommation - comme le voulait la commande.

\section{Des récits distincts qui intègrent plus ou moins le paysage}

Pourtant, une marge de liberté a été accordée aux cinéastes ; on peut repérer un certain nombre de partis pris personnels au-delà de ces similitudes générales. Pour la composition formelle et la narration, en particulier, des qualités et des différences apparaissent dans le nombre de plans et le détail de leurs enchaînements (les raccords visuels et sonores), le choix des genres musicaux (chants populaires traditionnels dans Bari et Napoli, musique classique d'opéra dans Milano, ou compositions originales pour Bologna, Firenze, Genova, Udine, notamment), la présence ou l'absence de voix off et d'images d'archives, ou encore les cadrages (majorité de plans serrés ou de plans larges). 
Planche 1. Photogrammes du film Bari de Lina Wertmüller (1989).

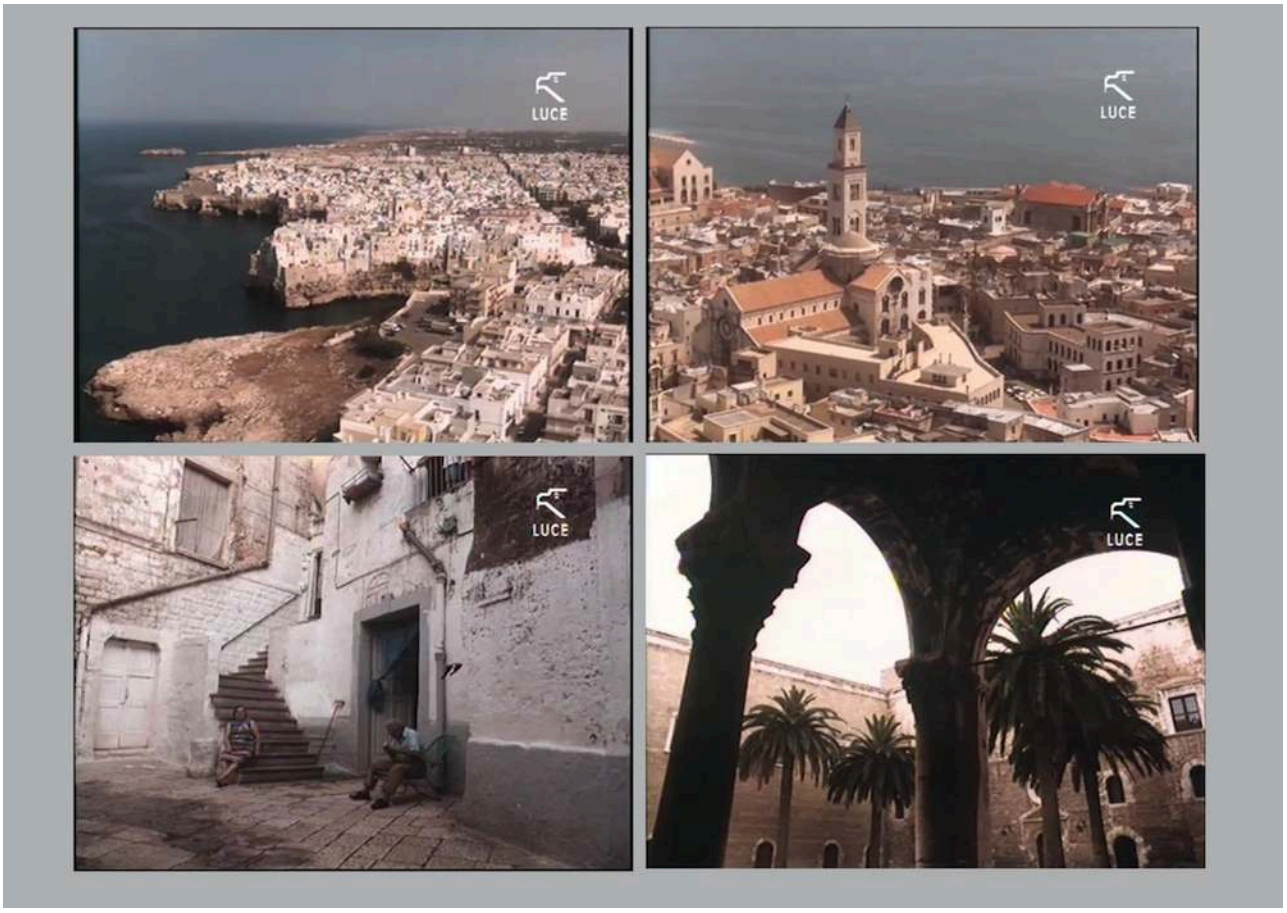

Des vues aériennes obliques, ménageant des horizons, côtoient des plans de rue et des cadrages s'appuyant sur l'architecture.

(c) Istituto Luce

Les propositions se démarquent davantage les unes des autres lorsqu'on observe en détail le contenu narratif des situations filmées, et l'analyse montre qu'il faut nuancer l'appréciation singulière de chaque séquence. Si Milano (Olmi) et Firenze (Zeffirelli) font figurer des acteurs en costume et accumulent les clichés, réduisant littéralement l'architecture de la ville à un décor de cinéma par les choix de localisation, d'éclairage et d'angles de vues, ces films mettent aussi en évidence le rôle déterminant du fleuve pour chacune de ces villes - tout comme Torino (Soldati) insiste sur le rôle du Pô, et Udine (Pontecorvo) sur celui du Tagliamento. Plusieurs autres courts-métrages comme Bari (Wertmüller), Napoli (Rosi) et Genova (Lattuada) associent des vues très conventionnelles du patrimoine architectural et urbain, proches de la carte postale, à des aperçus de l'animation quotidienne des rues et des places. Ces plans se rapprochent alors davantage du reportage ou du documentaire, montrant des usages ordinaires. Verona (Monicelli) s'appuie sur l'humour tout en employant des trouvailles narratives originales; des vues paysagères et des ralentis mettent en scène la légende du saint patron local, San Zeno, racontée de manière vivante et ludique (on voit même un personnage léviter dans les airs, ce survol de la ville justifiant en quelque sorte, visuellement, les plans tournés en vue aérienne). Ce montage éclectique associe la légende chrétienne, la fiction (Roméo et Juliette) et le patrimoine culturel national (Dante, Giotto, Pisanello, les monuments romains). Le réalisateur de Firenze, quant à lui, assume jusqu'au bout l'esthétique du clip vidéo et de la célébration: musique ample composée par Ennio Morricone, acteurs en costume, accumulation de ralentis et de travellings latéraux, scènes d'action, références continues au sport et au football via la tradition locale et spectaculaire du calcio storico $^{3}$. 
Dans certains cas, le récit se fait plus explicatif et plus précis sur le plan historique. Dans Cagliari, Lizzani fait le choix de commenter en voix off l'histoire de la ville, en repartant de son environnement et de ses vestiges archéologiques, mais aussi des rituels traditionnels montrés à l'écran, sur un mode didactique qui compense en partie le défilement rapide du montage. Lattuada fait de même pour Genova, comme Bolognini pour Palermo et Pontecorvo pour Udine ; tandis que Soldati, dans Torino, fait intervenir un personnage de guide.

Planche 2. Photogrammes du film Genova d'Alberto Lattuada (1989).

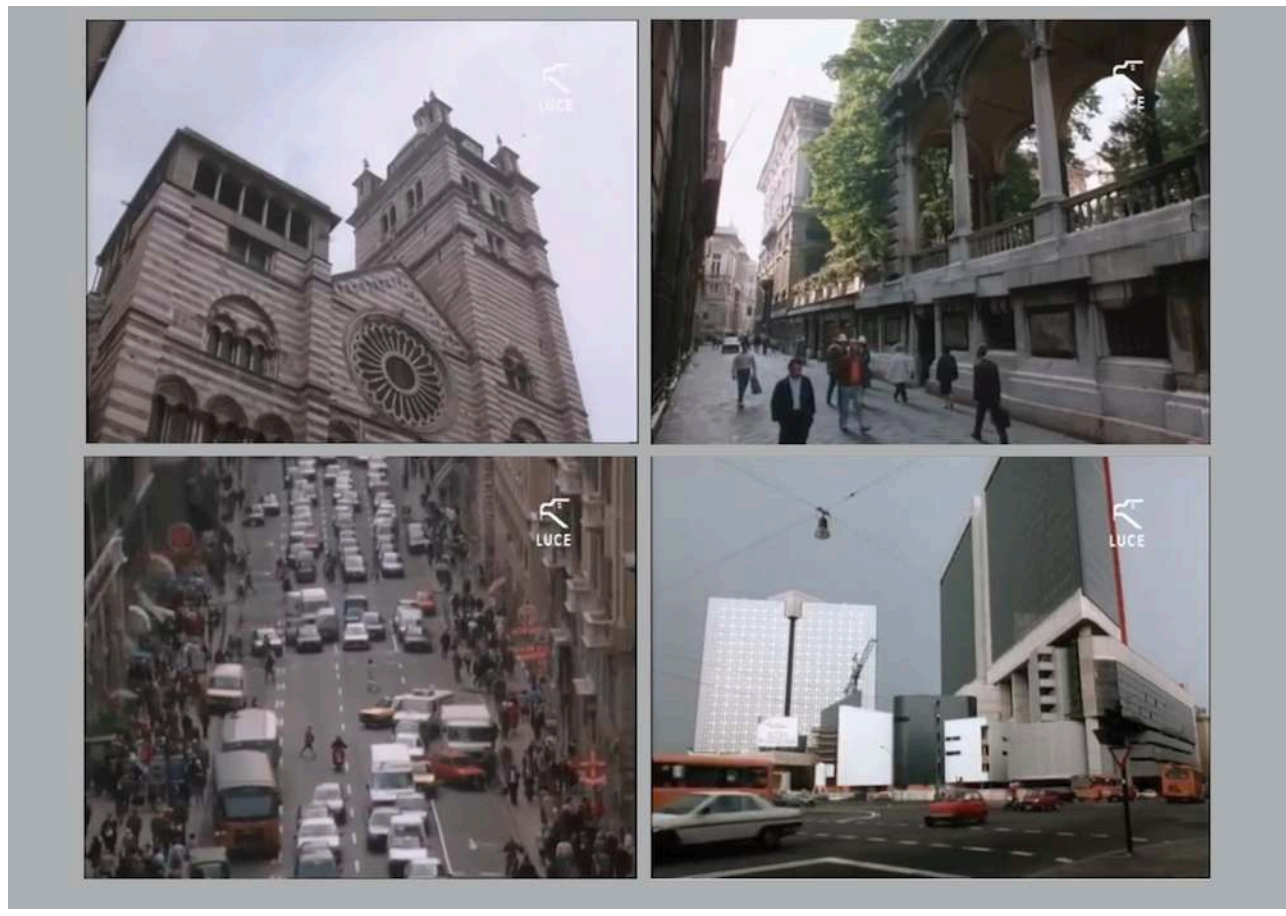

Le film fait apparaître différentes strates historiques de la ville, ainsi que certains aspects de sa vie quotidienne : le trafic automobile, les passants, l'animation des rues.

(c) Istituto Luce.

11 À la différence des autres séquences, Cagliari, Genova, Torino et Udine s'attardent sur les citadins et les espaces publics, les rues encombrées par le trafic, piéton et automobile. Bien que, de manière fugace, on aperçoive des personnes au travail; des sites de production industrielle; du linge étendu aux fenêtres. Comme les autres, ces films associent des vues de documents historiques en banc-titre ou en détail à des plans d'architecture de facture classique mais, à la différence des autres, ils accordent aussi une place significative à des éléments plus contemporains. Genova donne à voir les viaducs autoroutiers surélevés qui marquent si fortement le paysage de cette ville, et s'achève par de longs plans qui montrent l'emprise et les infrastructures de son immense port - certes associées à une musique enjouée. Torino livre un aperçu, en vue aérienne, des immenses usines FIAT de Mirafiori au sud de la ville (leur emprise monumentale occupe encore aujourd'hui deux millions de $\mathrm{m}^{2}$ ). Ce faisant, ces séquences semblent contourner, au moins en partie, les limites de la rhétorique touristique pour se rapprocher d'un style documentaire. Elles renouent du moins avec la tradition du portrait de ville en donnant à voir un contexte, une logique de complémentarité entre infrastructures productives et patrimoine artistique et 
architectural. Leurs auteurs accordent aux activités sociales de production, voire à l'environnement élargi, une visibilité qui tranche avec les choix de réalisation des autres films.

Planche 3. Photogrammes du film Genova d'Alberto Lattuada (1989).

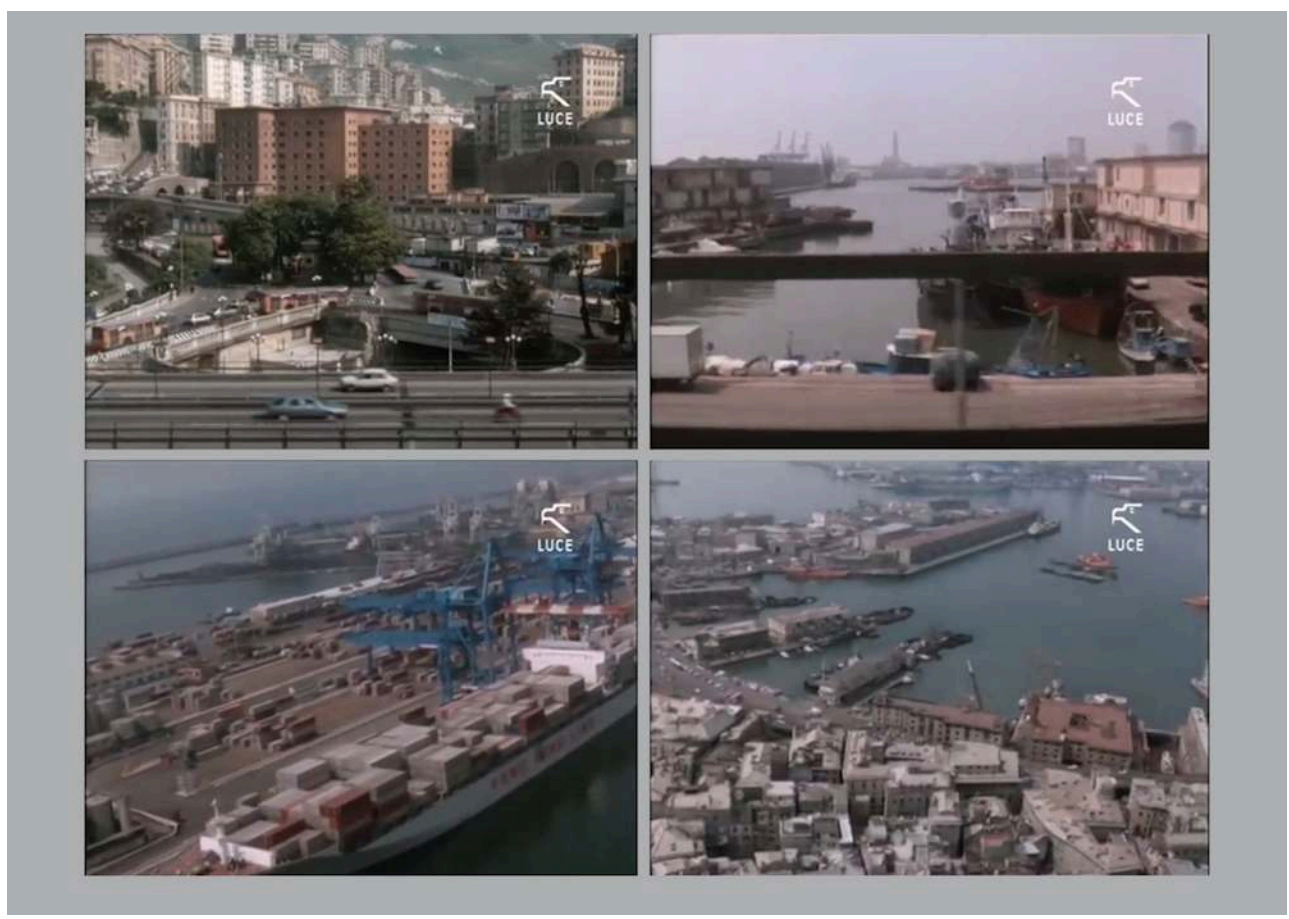

Comme les viaducs autoroutiers qui la traversent, les vastes infrastructures portuaires de Gênes sont des éléments caractéristiques du paysage de cette grande ville industrielle.

(c) Istituto Luce.

Cette attention se remarque aussi dans le regard porté sur l'eau, comme on l'a mentionné, sur le relief (Genova rappelle la topographie mouvementée de la ville et son rôle crucial dans l'histoire de son développement), et surtout sur les habitants euxmêmes. Bari rejoint ces trois séquences en cadrant aussi bien des visages de citadins en plan serré, au téléobjectif, que des vues d'autres villes et paysages des Pouilles, en particulier du littoral adriatique. Ce film-ci se distingue par la virtuosité de son montage, dont le rythme très soutenu s'accorde avec celui des accompagnements musicaux sans embrasser la logique d'un clip vidéo ; il compte plus de deux cents plans qui combinent des portraits d'enfants, d'adultes au travail, de personnes âgées assises sur le pas de leur porte, des cadrages d'architecture ainsi que des vues aériennes montrant des sites marquants du front de mer. Les villes filmées sont à la fois habitées et situées dans leur contexte paysager. 
Planche 4. Photogrammes du film Bari de Lina Wertmüller (1989).
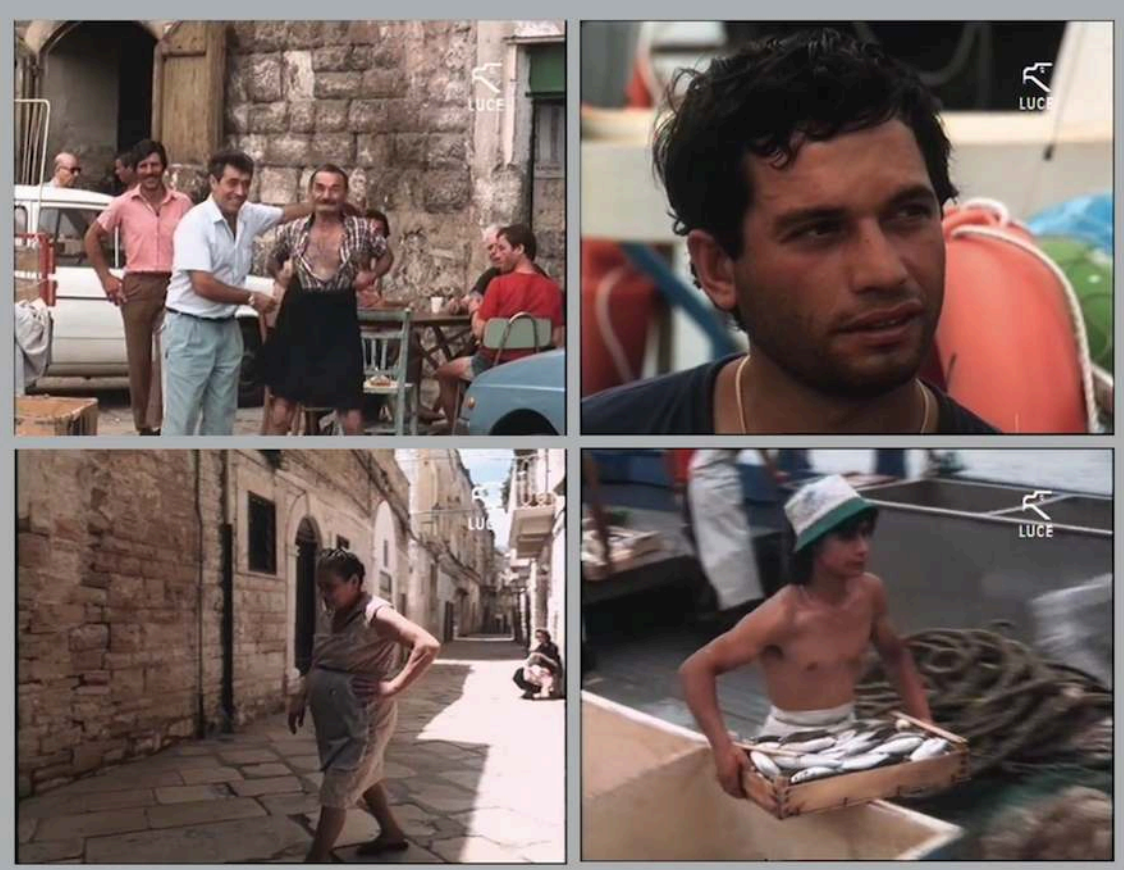

Le montage rapide de cette séquence accorde une place importante aux visages et aux mouvements quotidiens des citadins. Bari montre une ville habitée, faisant varier les focales, les cadrages et les angles de vue.

(c) Istituto Luce.

Napoli est tourné en majorité depuis le ciel: en multipliant les vues aériennes et les travellings, en cherchant en contre-plongée les cimes des immeubles, le montage réitère les raccords entre vues historiques et cadrages paysagers sur la baie, le Vésuve, les îles. Ces choix formels n'empêchent pas Rosi de suivre un ordre chronologique précis dans la séquence : de l'Antiquité au trafic automobile et aux gratte-ciel.

De manière comparable, Udine se démarque par une série de plans d'ouverture très picturaux sur les Alpes frioulanes. Outre que ces cadrages sur le milieu naturel constituent les images de paysages les plus classiques de toute la série, ils exposent de manière didactique, en suivant les cours d'eau le long des pentes, la relation entre l'implantation urbaine et la morphologie de l'environnement, à commencer par sa topographie et son hydrographie; leur succession, toute pédagogique, déploie une logique d'articulation des échelles spatiales. La progression du film élabore un sens de la mesure, comme y insiste la phrase de conclusion (« Udine, ville à mesure humaine ») dont le caractère de slogan publicitaire n'empêche pas la pertinence, le court-métrage en faisant la démonstration. Le récit historique et la visite architecturale sont appréhendés d'une manière originale: les musées, les palais et leurs fresques s'inscrivent dans un cadre géographique fait de pentes terrassées, de chemins suivant des rivières, de rues épousant le relief.

Ces trois derniers films, en particulier, opèrent donc une contextualisation pertinente et efficace, malgré les limites du format imposé. 


\section{Bologne en mouvement} les frères Bernardo et Giuseppe Bertolucci ${ }^{4}$, font des choix de réalisation subtils qui atteignent un haut degré d'invention et de poésie, par la combinaison de divers procédés cinématographiques et scénaristiques. La description détaillée du premier plan du film en rend compte, me semble-t-il, de manière suggestive.

17 Extérieur jour. Des taches de couleur et de lumière, une voix d'enfant. L'image est d'abord floue, le cadrage très serré gêne la mise au point. À mesure que la caméra s'éloigne, on distingue les cheveux puis le dos d'une petite fille. Elle est vêtue d'une robe rose et appuie son bras et sa tête contre un mur. Le travelling arrière fait apparaitre, de biais, un assemblage de pierres massives de couleur ocre, à la teinte éclaircie par la lumière matinale. La surface filmée en gros plan donne à voir l'usure et la patine irrégulière du mur mais aussi son "bossage rustique " très varié (bugnato rustico). Certains blocs sont arrondis, d'autres ornés (la fonction d'origine de ce travail de la pierre était à la fois défensive et décorative). "Novanta, novantuno, novantadue, novantatré... ${ }^{5}$ " Tandis que la petite fille finit de compter jusqu'à cent, le spectateur reconnait immédiatement un jeu familier. Se retournant vers la vaste place que l'on aperçoit désormais à gauche de l'image en plan large, elle lance le jeu de cache-cache : «Ch'è fuori è fuori, ch'è dentro è dentro ${ }^{6}$ " Dans une lumière de contre-jour produisant un effet de contraste et de clair-obscur, la caméra opère un mouvement panoramique vers la gauche. À l'image, apparaît la perspective d'une imposante colonnade dont les ouvertures accueillent des séries de trois larges marches; puis de hauts édifices crénelés, un fragment de façade de marbre blanc et rose sculpté, elle-même surélevée et qui paraît posée, à hauteur d'œil, sur un socle d'une volée d'une dizaine de marches. 
Planche 5. Photogrammes du film Bologna de Bernardo et Giuseppe Bertolucci (1989).

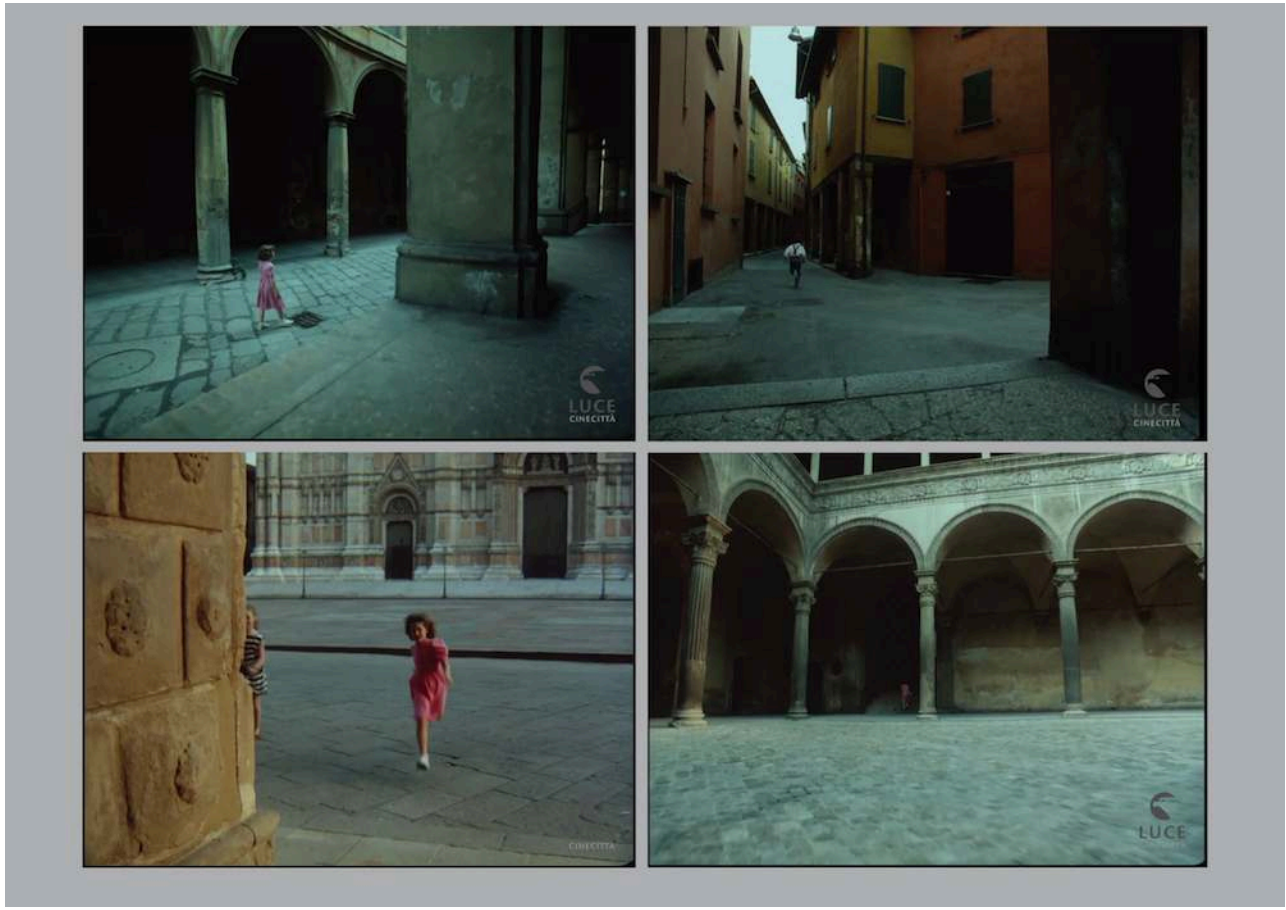

La séquence, très composée, place le regard des spectateurs à la hauteur de celui des enfants, et adopte le rythme de leurs déplacements et de leurs jeux dans les rues du centre historique.

(c) Istituto Luce.

Les huit minutes du film suivent les parcours de quelques jeunes enfants dans la ville silencieuse, presque entièrement vidée de ses adultes. Dans ce montage au rythme rapide, le mouvement est partout - course des enfants, travellings latéraux et circulaires, passage d'un vélo, successions de plans brefs - et presque toujours vu de biais, aussi proche du sol que le regard des protagonistes eux-mêmes. Le jeu qui recommence sitôt fini semble parfois tourner en rond, revenant à plusieurs reprises et sous un angle chaque fois différent au portique de la piazza Maggiore, cœur historique et géographique de la ville. Prétexte à une découverte de Bologne, la mise en scène de la partie de cache-cache est destinée à animer les bâtiments et les rues de son mouvement circulaire. Plusieurs lieux et monuments majeurs sont longés ou traversés, mais aucune vue d'ensemble frontale n'est retenue. La caméra s'attache plutôt à suivre les enfants le long des murs et des portiques, emprunte les escaliers, les cours et les passages ouverts, accompagne la petite fille qui pousse les battants de portes et de grilles. À l'image d'une séquence tournée dans le plus grand théâtre de la ville, c'est depuis les coulisses que nous entrons, in medias res, sur la scène de Bologne. Loin de s'arrêter pour la contempler, la caméra nous entraîne sous les portiques et dans les passages des rues étroites, si bien que chaque plan évoque une scène d'intérieur ${ }^{7}$. Outre le souffle rapide des enfants, on entend de temps à autre des chants d'oiseaux tandis que résonne de tous côtés le bruit des pas légers sur le sol. Mais aussi: des chuchotements et voix rieuses, une volée de cloches, puis quelques notes d'une discrète musique de fond, intermittente, d'instruments à vent et à cordes pincées ou frottées. Autant d'effets sonores qui accentuent l'effet d'immersion et d'enveloppement. Le film s'achève sur un plan nocturne reprenant exactement le même cadrage, le même texte et le même mouvement d'appareil que le plan d'ouverture, sauf qu'un jeune garçon a 
remplacé la petite fille et qu'un orchestre d'une vingtaine de musiciens, un peu esseulé dans le champ et que le regard de la caméra ne suit pas, traverse la place déserte en jouant L'Internationale (Bologne était encore, à l'époque du tournage, l'un des principaux bastions communistes de la péninsule, et ce depuis 1945).

Ce traitement subtil n'empêche pas les deux réalisateurs d'intégrer quelques clichés relevant d'une imagerie caractéristique de la ville, que tout visiteur pourra identifier dès sa première journée de découverte : la statue du Neptune, le théâtre anatomique du bâtiment de l'Archiginnasio, le bibelot produisant de la neige, ou encore la marionnette du truculent Dottor Balanzone qui s'exprime en dialecte local ${ }^{8}$. Le sujet lui-même, la course des enfants dans le centre-ville historique, peut être qualifié de banal et se retrouve dans bien des déclinaisons commerciales, comme le montrerait sans difficulté une étude plus attentive du rôle des enfants dans les publicités immobilières ou touristiques. L'originalité tient ici à l'angle retenu et à la progression du récit: à l'exception de deux brefs plans ${ }^{9}$, la narration non dialoguée se tient tout au long de la séquence à hauteur du regard des enfants et adopte le rythme de leur propre déplacement dans les rues de la ville. Parce qu'elle est tenue d'un bout à l'autre, sans commentaire ni épilogue, cette simplicité stylistique produit en fin de compte un effet d'animation de la ville, qui tranche avec les onze autres films. L'artifice de l'avoir vidée de ses passants et activités, loin de la figer dans un pesant sommeil, devient l'occasion d'accompagner son réveil au fil d'un jeu d'enfants en mouvement. Jeu de poursuite et d'attente, d'excitation et de fuite, le cache-cache se fait l'instrument d'une découverte de la morphologie urbaine singulière de Bologne : ses rues tournantes qui embrassent les détails de la microtopographie du sol, et surtout ses innombrables portiques (plus de quarante kilomètres) qui offrent d'infinies possibilités de cachettes et de surprises. L'expérience esthétique que procure cette ville n'est pas un majestueux spectacle visuel composé d'alignements perspectifs vers le lointain, de façades aux ornements splendides ou aux dimensions saisissantes. Elle est faite d'ombres et de silences. Elle n'en est que plus riche et consistante. Cette extraordinaire qualité d'expérience ne se comprend que par le mouvement : seule la marche met en évidence l'articulation des échelles et des usages divers de ces formes urbaines singulières, caractérisées à la fois par leur cohésion et leur polyvalence. C'est de tout ce mouvement, corporellement ressenti, que le court-métrage des Bertolucci parvient à rendre compte.

\section{L'imagerie culturelle du paysage urbain à l'écran : industrie du tourisme, commande d'État ou immersion documentaire?}

Cette analyse comparative, bien que non exhaustive, met en évidence l'originalité de cette série de courts-métrages. On pourrait certes engager une discussion critique plus poussée sur les critères qui conduisent à justifier la notion de paysage urbain, séquence après séquence. J'espère néanmoins avoir montré que ces vues urbaines très composées entretiennent un rapport précis avec la tradition picturale des portraits de ville, ainsi qu'avec celle des vues paysagères. Tout en reprenant les codes esthétiques de ces imageries spécifiques, chacun des films cités les renouvelle à sa manière, de façon plus ou moins audacieuse et subtile. Faisant mieux percevoir leurs différences, leur juxtaposition en une même série compose un ensemble remarquable par la diversité des choix narratifs et de discours. 


\section{L'imagerie sans le paysage : deux cas limites}

21 serie, revenons brièvement sur deux d'entre eux. Comme la plupart des autres films, Palermo (Bolognini) et Roma (Antonioni) accordent une attention méticuleuse au patrimoine artistique et architectural ; mais à la différence des autres, ils s'y limitent exclusivement, au point d'en éclipser les citadins eux-mêmes ${ }^{10}$. Cette exclusion est d'autant plus visible qu'elle tranche avec les autres séquences. Le cas de Roma ne peut susciter qu'une certaine déception chez l'amateur de cinéma. À contre-pied de ses propres œuvres, Antonioni s'absorbe longuement dans les fresques du Vatican, l'architecture baroque de l'intérieur des palais, deux plans de ponts sur le Tibre et un bref panorama, lointain, de la Ville éternelle, sous un ciel chargé de sombres nuages. Pour une raison ou une autre, il semble que le réalisateur n'ait pas voulu s'emparer de la commande, au point de livrer un court-métrage très distant et impersonnel. En faisant disparaître la ville au profit d'une sélection d'œuvres sculpturales et surtout picturales (plutôt que de monuments dans des lieux publics, par exemple), il fait également abstraction du paysage, pourtant si caractéristique et si célébré de Rome. La plupart des plans sont fixes et tournés en intérieur. Ils se concentrent sur l'expressivité d'une sélection d'œuvres plastiques certes remarquables, mais si célèbres qu'on peut penser qu'une large majorité des spectateurs les auront déjà vues, au moins en reproduction, sous une forme ou une autre. Le film n'intègre en outre aucune sorte de commentaire, et s'achève... sur le centre de la fresque de Michel-Ange au plafond de la chapelle Sixtine. Que penser de ces choix étonnants, pour un tel cinéaste? Quelles qu'en soient les motivations, ils ont pour résultat la production d'une imagerie extrêmement conventionnelle et statique. Ces remarques valent aussi pour Palermo, qui se concentre sur des visites de lieux clos, d'intérieurs et de monuments coupés de leur contexte, ou encore de cloîtres déserts, sans jamais montrer le ciel, le littoral, les collines qui entourent le chef-lieu de la Sicile, ni l'animation si caractéristique de ses rues et ruelles - hormis une très brève scène pittoresque de marché, en plan relativement serré, qui ne dure que quelques secondes; et encore est-ce, de manière révélatrice, pour la rapporter à l'œuvre du peintre Renato Guttuso.

En fin de compte, Palermo et Roma recomposent un album culturel entièrement introverti, au plus loin de toute intention d'immersion documentaire. Il s'agit d'une iconographie abstraite et coupée de la vie urbaine, encombrée de chefs-d'œuvre que les mouvements de caméra cherchent à animer. Ce sont ainsi, à mes yeux, des cas limites où la prépondérance de l'imagerie officielle $d u$ (ou d'une partie du) patrimoine artistique en vient à effacer la possibilité d'une représentation paysagère de la ville.

Ces deux films rappellent au spectateur sa condition de touriste, sans ironie. Par leur médiation, nous admirons à distance les éléments d'une imagerie très contrôlée qui, pour rester conventionnelle, n'en dégage pas moins une émotion et une attraction. D'une certaine manière, l'attitude des réalisateurs est ici de chercher à se mettre au service des œuvres et de leurs commanditaires. Dans un cas aussi politique que Rome et le Vatican (mais Palerme fut elle aussi une capitale à part entière pendant plusieurs siècles, comme la majorité des douze villes montrées dans la série), l'effacement du cinéaste devant l'autorité des œuvres suggère une sorte d'allégeance. Cette attitude rappelle incidemment que la réalisation des films émane d'une commande d'État. S'interroger sur l'identité et les finalités des commanditaires d'une œuvre d'art est

Projets de paysage, 19 | 2018 
d'autant plus pertinent dans le cas de l'Italie et du Vatican que la plupart des œuvres et du patrimoine architectural et urbain du pays proviennent de ce type de démarche. Aucune des œuvres montrées dans Roma n'échappe à cette logique de servir le pouvoir. Des papes de la Renaissance à la période fasciste, Rome n'est-elle pas restée un foyer de «l'éloquence architecturale " affirmée dès le Quattrocento dans les cités princières du Nord de l'Italie (Boucheron, 2014) ? Comme l'ont montré les historiens, la production d'œuvres d'art relève, dans un tel contexte, de l'exécution d'un programme politique de prestige - voire de propagande pure et simple - destinée à impressionner le visiteur étranger (l'ambassadeur, le pèlerin, le touriste). L'artiste se met au service d'une fabrication politique des images, que notre regard contemporain pourrait avoir tendance à négliger ou à oublier. C'est l'un des intérêts des films sur Palerme et sur Rome que de le remettre en évidence.

\section{Le tourisme, une économie d'État fondée sur la production d'images de biens publics}

24 À l'occasion de l'organisation de la Coupe du monde du football par l'Italie, une commande étatique semble donc avoir associé l'industrie culturelle et artistique du cinéma avec celle du tourisme. Il serait absurde de reprocher à des œuvres de commande à finalité commerciale de remplir leur cahier des charges, au service du marché des grands événements sportifs internationaux. Trente années après la réalisation de ces films promotionnels, l'usage des images par et pour le tourisme a changé de forme et d'ampleur, mais non de principe. En Italie et en France, en particulier, il n'est pas exagéré de tenir le développement systématique du tourisme et de ses infrastructures pour une véritable économie d'état. Cette remarque ouvre un certain nombre d'interrogations sur le statut marchand ou commercial du patrimoine collectif et des biens publics. Même rapportée au cadre contraignant d'une commande, la mise en scène d'un paysage urbain par un artiste engage une conception implicite du «patrimoine culturel public» (Settis, 2002), voire de l'espace public urbain comme image de la vie collective (Joseph, 1998). Mais en l'occurrence, il s'agit aussi de prendre part à la compétition économique que représente le tourisme de masse, dans des sociétés où l'industrie productive est en recul ; au risque, selon certains observateurs et dans des cas aussi extrêmes que celui de Venise, de tendre à perdre le sens de l'appartenance et de la participation effective à la vie collective d'une ville (Settis, 2015). À l'inverse, les séquences en plans rapprochés de la population et de l'architecture vernaculaire peuvent, à la manière d'un documentaire social, suggérer un effet d'immersion qui tranche avec le regard consumériste du tourisme de masse. Les différences les plus significatives, d'un film à l'autre de la série, se jouent sur de telles lignes de partage. Il ne s'agit pas d'une simple différence d'esthétique, mais d'une divergence plus profonde, relevant d'implications éthiques, politiques et épistémologiques - de la «culture visuelle» au sens proposé par l'historien W. J. T. Mitchell (2014, dernier chapitre) - et que l'on pourrait apprécier en détail, plan par plan, dans la manière de chaque cinéaste.

Rappeler le contexte social et culturel de la production de ces films permet de mieux en apprécier le sens et la portée. À la fin des années 1980, la logique promotionnelle de l'imagerie touristique qui s'est imposée depuis dans la représentation officielle -c'est-àdire issue de commandes et de subventions publiques - des villes était encore loin d'avoir pris sa forme actuelle de «marketing » urbain, fondée sur la compétition entre 
les destinations touristiques à l'échelle européenne, puis mondiale. Le degré d'uniformisation et d'aseptisation des représentations urbaines contemporaines saute aux yeux lorsqu'on regarde, en comparaison, cette série cinématographique de 1989. Rétrospectivement, les années 1990 et 2000 auront été le théâtre de l'élaboration puis de la généralisation de cette vocation marchande de la fabrication d'images urbaines sur des fonds publics, notamment en marge d'autres événements sportifs de masse tels que les jeux olympiques.

\section{Représenter la géographie culturelle des villes italiennes}

Douze réalisateurs pour douze villes: le titre même de la série annonçait déjà que la commande laissait aux auteurs la liberté de se positionner vis-à-vis de celle-ci, de la reformuler en partie et de l'interpréter. En majorité, les films ont bel et bien proposé des regards particuliers, voire personnels. Aucun de ces portraits de ville, certes, n'échappe à l'anecdote ou au folklore et à ses limites - à l'image de Napoli se refermant sur le personnage traditionnel de Pulcinella (Polichinelle), esseulé sur la scène d'un théâtre éclairé mais désert. Ils reprennent tous de manière plus ou moins appuyée l'imagerie traditionnelle de l'Italie vue de l'étranger: du Grand Tour au petit écran, en passant par la carte postale, voire le kitsch (le cadrage de la baie de Naples avec le Vésuve), et par les fresques de la chapelle Sixtine. Pourtant, on aurait tort de négliger l'originalité du travail de chacun des douze réalisateurs et, dans la plupart des cas, la réflexion qu'ils ont engagée sur le sens de cette imagerie culturelle des portraits urbains. Une vue d'ensemble confirme qu'ils ont su produire davantage qu'une série statique d'arrêts sur image ou qu'un égrenage de clichés. Au fil des paysages aperçus, tel ou tel film de la série - en particulier Bari et Bologna - ouvre même la possibilité d'une brève immersion dans l'héritage vivant d'une localité. Cette démarche s'oppose à l'idéalisation et incite à adopter un point de vue critique sur les autres séquences. Les cinéastes, en répondant à la commande par de petits films d'auteur personnels et attentifs aux espaces ordinaires des villes choisies, s'affranchissent d'une esthétique conventionnelle sans la contredire frontalement. La subtilité de leur démarche tient à une approche du paysage urbain que l'on pourrait qualifier de culturelle, voire d'anthropologique dans la mesure où elle suggère des liens, des attaches et des attachements affectifs et sociaux. Les Bertolucci à Bologne, comme Lina Wertmüller à Bari et, dans une moindre mesure, Carlo Lizzani à Cagliari et Alberto Lattuada à Gênes s'efforcent de transmettre au spectateur une relation de participation émotionnelle avec les lieux habités, en travaillant des clichés visuels de l'intérieur. Ils esquissent une idée sensible des villes concernées, en quelques minutes d'un montage serré.

«C'est ainsi que l'on devrait considérer les paysages : pas seulement en fonction de leur apparence ou de leur conformation à tel ou tel idéal esthétique, mais aussi d'après leur façon de satisfaire les besoins élémentaires, comme celui de partager certaines de ces expériences sensorielles dans un endroit familier : des chansons populaires, des plats populaires, une espèce particulière de climat censée ne se trouver nulle part ailleurs, un sport ou un jeu spécial, qui ne se pratiquerait qu'à cet endroit. Ces choses nous rappellent que nous sommes, ou que nous venons, d'un lieu particulier : un pays, une ville, un quartier. Un paysage devrait établir des liens entre les gens, le lien que créent la langue, les manières, la pratique du même genre de travail ou de loisir, mais surtout un paysage devrait contenir le genre d'organisation spatiale qui favorise ces expériences et ces relations: des espaces pour se rassembler, pour célébrer, et des espaces pour la solitude, des espaces qui ne changent jamais et demeurent toujours tels que la mémoire nous les dépeint. Ce 
sont là quelques-unes des caractéristiques qui donnent à un paysage son côté unique, qui lui donnent un style, et qui font que nous nous le rappelons avec émotion. » (Jackson, 2005, p. 42) Ces publicités réalisées par de grands cinéastes procèdent parfois de choix esthétiques qui les distinguent de la logique spectaculaire et marchande du tourisme de masse alors même qu'elles y participent. Si leur valeur esthétique est relative et peut être contestée, la série échappe à la caricature ou au poncif dicté par les impératifs commerciaux. Cette analyse comparée a mis au jour la diversité de style et de contenu des courts-métrages, ainsi que l'enrichissement de points de vue qui procède d'une telle juxtaposition : l'exercice de représentation cinématographique, grâce à des choix techniques maîtrisés, peut déjouer les limites d'une commande commerciale. L'observation, l'imagination et le savoir-faire élargissent le spectre d'une imagerie typique, indexée à des modèles statiques tels que les cartes postales ou les brochures des agences de voyages. En d'autres termes, à condition que la liberté lui en soit laissée, l'artiste est capable de reformuler la commande publicitaire qui lui est faite sans pour autant lui faire défaut. Il n'est pas surprenant que l'imagerie commerciale donne lieu à des expérimentations formelles, renouvelant les conventions du genre; les plasticiens des avant-gardes des années 1920 l'avaient déjà montré. Il est plus remarquable qu'une telle commande, dans un cadre aussi contraint que celui de la promotion d'une Coupe de monde de football, ait pu laisser advenir des représentations plus subtiles, donnant à voir les aspects géomorphologiques des paysages, les visages de citadins anonymes et les espaces publics fréquentés, voire les infrastructures industrielles, de plusieurs villes italiennes.

Leur étude pourrait aussi contribuer à nuancer notre appréciation critique de l'expérience touristique contemporaine, comme y invitait Jackson lorsqu'il relevait la 
"dimension éducative du tourisme dans la plupart de ses formes» (2005, p. 25) l'auteur lui-même allant jusqu'à se présenter comme un "touriste professionnel ». L'ampleur quantitative et les conditions qualitatives du tourisme ont certes beaucoup changé depuis cette affirmation. Cette croissance spectaculaire appelle une réflexion approfondie sur nos manières de représenter les villes et les paysages, ainsi que sur la circulation de cette imagerie au sein des réseaux de communication contemporains. Cette série de portraits urbains filmés est en mesure d'inspirer les études de paysage aujourd'hui : elle montre qu'une même finalité, la représentation touristique dans le cadre d'une commande publicitaire, peut donner lieu à une pluralité d'interprétations artistiques attentives aux enjeux esthétiques, culturels et anthropologiques de nos expériences de la ville et des paysages urbains.

\section{BIBLIOGRAPHIE}

Boucheron, P., De l'éloquence architecturale. Milan, Mantoue, Urbino, 1450-1520, Paris, Éditions B2, 2014.

Chabrol, C., Godard, J.-L., Rivette, J., Rohmer, É., et Truffaut, F., La Nouvelle Vague, textes, réunis par Antoine de Baecque, Gabrielle Lucantonio et Charles Tesson, Paris, Cahiers du cinéma, coll. «Petite anthologie des Cahiers du cinéma », 1999.

Debarbieux, B., « Actualité politique du paysage », Revue de Géographie Alpine | Journal of Alpine Research, 95-4, 2007, mis en ligne le 24 juin 2009, URL : http://journals.openedition.org/rga/382.

Flusser, V., Into the universe of technical images, Minneapolis, University of Minnesota Press, 2011.

Gervereau, L., Histoire du visuel au XX $x^{e}$ siècle, Paris, Éditions du Seuil, 2003.

Ingold, T., " The Temporality of the Landscape », The Perception of the Environment: Essays on Livelihood, Dwelling, Skill, Londres, Routledge, 2000.

Jackson, J. B., « À l'école des paysages », De la nécessité des ruines et autres sujets (1980), traduit de l'américain par S. Marot, Paris, Éditions du Linteau, 2005.

Jacques, V., Chris Marker, les médias et le $\mathrm{XX}^{e}$ siècle : le revers de l'histoire contemporaine, Ivry, Créaphis, 2018.

Joseph, I., « Paysages urbains, choses publiques », Les Carnets du paysage, n 1, 1998, Dossier « Le paysage comme espace public », p. 70-88.

Jousse, T. et Paquot, T., La Ville au cinéma, Paris, Les Cahiers du Cinéma, 2005.

Malpas, J., The Place of Landscape: Concepts, Contexts, Studies, Cambridge, MIT, 2011.

Mitchell, W.J.T., Que veulent les images? Une critique de la culture visuelle, Dijon, Les Presses du réel, 2014.

Ricci, G., Bologna, Roma/Bari, Laterza, 1980.

Settis, S., Si Venise meurt, traduit de l'italien J. Pietri, Paris, Hazan, 2015.

Settis, S., Italia S.p.A. L'assalto al patrimonio culturale, Turin, Einaudi, 2002. 


\section{NOTES}

1. Dont l'archétype est Berlin, symphonie d'une grande ville de Walter Ruttmann (1927), fresque en mouvement illustrant une journée de Berlin grâce à des prouesses de montage. Pour d'autres exemples, voir notamment Jousse et Paquot (2005).

2. Ce film de 15 minutes met en scène le réalisateur lui-même tenant un discours très articulé sur l'évolution contemporaine des silhouettes des villes italiennes. Il a été tourné à Orte et à Sabaudia en 1974 pour le compte de la télévision nationale (RAI).

3. Le "football historique " est un jeu de ballon florentin à mi-chemin entre le rugby et le football modernes, caractérisé par un degré d'engagement physique très prononcé : il implique des confrontations violentes entre les joueurs (coups, crochetages, saisies, plaquages, voire affrontements directs). Il donne lieu à un tournoi annuel sur la grande place de Santa Croce, très prisé de la population locale.

4. Les deux frères étaient cinéastes bien que l'aîné, Bernardo (1941-2018), soit plus célèbre. Giuseppe Bertolucci (1947-2012) a réalisé plus d'une trentaine de films et collaboré avec son frère en tant que scénariste pour différents projets. Les deux frères, natifs de Parme, avaient un attachement personnel pour la ville de Bologne, qu'ils connaissaient parfaitement pour y avoir étudié ou séjourné fréquemment.

5. «Quatre-vingt-dix, quatre-vingt-onze, quatre-vingt-douze, quatre-vingt-treize... » Il n'est évidemment pas indifférent que le film soit tourné en 1989 pour célébrer un événement national en 1990 ; le décompte du personnage se projette vers le nombre des années à venir, suggérant au passage que l'âge vénérable de Bologne n'altère nullement sa capacité à se renouveler, à rester jeune.

6. Littéralement: "Qui est dehors est dehors, qui est dedans est dedans!», expression idiomatique italienne dont l'équivalent français pourrait être : «Les jeux sont faits, rien ne va plus!» Et qui, en l'occurrence, s'entend aussi comme un jeu sur la description littérale des espaces spécifiques de Bologne, dont les portiques entremêlent de manière extraordinaire les espaces intérieurs et extérieurs.

7. À l'exception notable d'une brève vue aérienne, toujours en mouvement, du sanctuaire de San Luca, et d'un plan panoramique nocturne depuis le belvédère de San Michele in Bosco, au sud de la ville. Ces deux plans apportent un élément d'équilibre et une ouverture vers les représentations les plus conventionnelles du paysage de Bologne.

8. Bologne aurait légué ce personnage de savant ridicule à la commedia dell'arte. Il préfigure très explicitement les médecins pédants de Molière.

9. Les deux vues citées ci-dessus, dans la note 7 .

10. Sauf quelques très brefs plans d'un marché de Palerme, dans le film de Bolognini, eux-mêmes associés par le montage à des tableaux du peintre sicilien Renato Guttuso (1912-1987).

\section{RÉSUMÉS}

12 Registi per 12 città (1989) est un film de commande promotionnel composé de douze portraits de villes, réalisés par douze cinéastes italiens de renom avant la $14^{\mathrm{e}}$ Coupe du monde de football (Italia '90). Il offre un corpus exemplaire pour étudier le rapport entre la représentation du paysage urbain et l'imagerie publicitaire. Comment sont représentées les villes? Que montrent ces mises en scène qui suivent une logique commerciale, touristique et patrimoniale ? L'article 
examine les places respectives que les films accordent aux vues paysagères et aériennes, aux monuments historiques ou aux caractéristiques des sites (le relief, l'eau) ; mais aussi l'attention variable que les différents cinéastes accordent aux lieux publics, aux environnements sonores, aux activités ordinaires et aux habitants de ces villes. Ces films publicitaires d'une grande maîtrise technique procèdent de choix esthétiques qui les distinguent parfois de la logique spectaculaire et marchande du tourisme de masse, alors même qu'ils y participent. Leur analyse permet d'affiner notre appréciation de la production d'images des paysages urbains et, au-delà, de l'expérience touristique.

12 Registi per 12 città (1989) is a commissioned promotional film comprising twelve portraits of cities, produced by twelve famous Italian filmmakers before the 14th World Cup (Italia'90). It is an exemplary body of works which can be used to study the relationship between the representation of the urban landscape and the advertising image. How are cities represented in these films? What do these portrayals, which follow a logic based on the market, tourism and the heritage, demonstrate? The article examines the respective roles given in these films to landscapes, aerial views, historical monuments, and specific elements within the sites (relief, water); it also takes a look at variations in the attention focused by the filmmakers on the public places, the sonic environments, the inhabitants and the day-to-day activities in these cities. These technically sophisticated commercial films are the result of aesthetic choices that sometimes distinguish them from the spectacular and commercial rationale of mass tourism which they nevertheless are a part of. Analysing them makes it possible to fully appreciate the production of urban images and the tourist experience.

\section{INDEX}

Mots-clés : paysage urbain, publicité, cinéma, représentation, tourisme

Keywords : urban landscape, advertising, cinema, representation, tourism

\section{AUTEUR}

\section{OLIVIER GAUDIN}

Olivier Gaudin, docteur en philosophie des sciences sociales (CEMS/EHESS), enseigne à l'École de la nature et du paysage de Blois (INSA CVL). Ses recherches portent sur le pragmatisme philosophique, l'écologie humaine en sciences sociales, et l'histoire culturelle des paysages. Il est directeur de la publication des Cahiers de l'École de Blois et membre de la rédaction des revues Métropolitiques et Pragmata. olivier.gaudin[at]insa-cvl[dot]fr

Page Academia : https://insa-centrevaldeloire.academia.edu/OlivierGaudin 